\title{
Electrospun Hyaluronan-Gelatin Nanofibrous Matrix for Nerve Tissue Engineering
}

\author{
Hau-Min Liou, ${ }^{1}$ Lih-Rou Rau, ${ }^{1}$ Chun-Chiang Huang, ${ }^{1}$ Meng-Ru Lu, \\ and Fu-Yin Hsu, ${ }^{1,2}$ \\ ${ }^{1}$ Institute of Bioscience and Biotechnology, National Taiwan Ocean University, Keelung 20224, Taiwan \\ ${ }^{2}$ Department of Life Sciences, National Taiwan Ocean University, Keelung 20224, Taiwan
}

Correspondence should be addressed to Fu-Yin Hsu; fyhsu5565@gmail.com

Received 23 July 2013; Revised 25 September 2013; Accepted 26 September 2013

Academic Editor: In-Kyu Park

Copyright (C) 2013 Hau-Min Liou et al. This is an open access article distributed under the Creative Commons Attribution License, which permits unrestricted use, distribution, and reproduction in any medium, provided the original work is properly cited.

\begin{abstract}
Schwann cells play a critical role in the repair of the peripheral nerve. The goal of this study was to fabricate electrospun gelatin (Gel) and hyaluronan-gelatin (HA-Gel) composite nanofibers to provide a suitable growth environment for Schwann cells. The fiber diameters of Gel, $0.5 \mathrm{HA}-\mathrm{Gel}, 1 \mathrm{HA}-\mathrm{Gel}$, and $1.5 \mathrm{HA}$-Gel were $130 \pm 30 \mathrm{~nm}, 294 \pm 87 \mathrm{~nm}, 362 \pm 129 \mathrm{~nm}$, and $224 \pm 54 \mathrm{~nm}$, respectively. The biological performance of Gel and HA-Gel was evaluated using an in vitro culture of RT4-D6P2T rat Schwann cells. We found that the cell attachment and proliferation rates were not significantly different on these matrices. However, the Schwann cells displayed better organized F-actin on HA-Gel than on Gel. Moreover, the expression levels of several genes, including Nrg1, GFAP, and P0, were significantly higher on HA-Gel than on Gel. In addition, the levels of Nrg1 and P0 protein expression were also higher on the HA-Gel than on Gel. These results indicate that the hyaluronan-gelatin composite nanofibrous matrix could potentially be used in peripheral nerve repair.
\end{abstract}

\section{Introduction}

Regeneration of the nervous system is a major challenge. However, the peripheral nervous system has a better intrinsic ability to repair and regenerate axons after axonal damage due to the ability of Schwann cells to enhance the environment for regeneration [1]. For the treatment of peripheral nerve injury, nerve autografts or direct end-to-end surgical reconnection is used to repair nerve injury. Unfortunately, direct end-to-end surgical reconnection only can repair small gaps in the nerve, because the tension introduced into the nerve cable would inhibit nerve regeneration for longer nerve gaps [2]. A nerve autograft harvested from another site in the body is used in these cases and is considered the "gold standard" for repair of the longer nerve damage gaps without immunological rejection problems. However, a nerve autograft has several disadvantages, including neuroma, hypofunction at the donor nerve graft site, and nerve site mismatch. Allografts and xenografts are possible replacements for autografts. Nevertheless, the major clinical problems are the risk of immune repulsion and disease transmission [3]. Thus, a nerve graft made of natural and synthetic materials is a promising alternative for promoting successful nerve regeneration.

Gelatin is obtained by the partial hydrolysis of native collagen in an alkaline or acidic environment. Gelatin exhibits excellent biocompatibility and biodegradability properties; thus, it has been widely used for nerve repair [4]. Hyaluronan is a naturally biopolymer composed of repeating disaccharide units of glucuronic acid and $\mathrm{N}$-acetylglucosamine. It is the major constituent of the extracellular matrix (ECM) of connective tissues and has many important biological functions. Because of its excellent biocompatibility, nonimmunogenicity, and specific biological functions, hyaluronan is used in a variety of clinical therapies, including supplementing joint fluid in arthritis and facilitating wound healing and regeneration $[5,6]$. Furthermore, hyaluronan is known to prevent perineural scar formation, which improves peripheral nerve regeneration [7].

Tissue engineered scaffolds are another choice for implantation to facilitate neural repair. Fibrous scaffolds have become very popular in tissue engineering because they mimic the physical architecture of the ECM. The architecture 
TABLE 1: Oligonucleotide primer for real-time PCR amplification.

\begin{tabular}{lc}
\hline Gene & Primer sequence: sense/antisense \\
\hline \multirow{2}{*}{ GFAP } & $5^{\prime}$-GGTGTGGAGTGCCTTCGTAT-3' \\
& $5^{\prime}$-TACGATGTCCTGGGAAAAGG-3' \\
NGF & $5^{\prime}$-CCAAGCACTGGAACTCATACTGC-3' \\
& $5^{\prime}$-CTGCTGAGCACACACACGCAG-3' \\
Nrg1 & $5^{\prime}$-GGCAGTCAGCCCCTTTGTG-3' \\
& $5^{\prime}$-TGCAGGGTTGTGATGAAAGGA-3' \\
p75 & $5^{\prime}$-CATCTCTGTGGACAGCCAGA-3' \\
& $5^{\prime}$-CTCTACCTCCTCACGCTTGG-3' \\
P0 & $5^{\prime}$-CTGCACTGCTCCTTCTGGT-3' \\
& $5^{\prime}$-CCTTGGCATAGTGGAAGATTG-3' \\
S100 & $5^{\prime}$-ATAGCACCTCCGTTGGACAG-3' \\
& $5^{\prime}$-TCGTTTGCACAGAGGACAAG-3' \\
18S rRNA & $5^{\prime}$-GGCCCGAAGCGTTTACTT-3' \\
& $5^{\prime}$-CGGCCGTCCCTCTTAATC-3' \\
\hline
\end{tabular}

of the ECM plays an important role in regulating cell behavior with biochemical signals and topographical cues $[8,9]$.

Electrospinning is an easy method to fabricate ultrafine fibers with submicrometer to nanometer diameters. Electrospun fibrous matrices exhibit certain special characteristics, such as a high specific surface area, a high aspect ratio, and a high porosity surface area. Most importantly, the topological structure of electrospun matrices mimics the architecture of the ECM and could enhance cell attachment, migration, proliferation, and differentiation [10].

Schwann cells are a major component of the peripheral nerve system and play an important role in Wallerian degeneration and the subsequent axon regeneration in peripheral nerve injury [11]. After Wallerian degeneration, the Schwann cells begin to proliferate and migrate to the lesion, forming longitudinally oriented cell strands called the bands of Büngner; thereafter, Schwann cells secrete a number of neurotrophic factors such as nerve growth factor and cytokines that support the survival of injured neurons [12], secrete extracellular matrix components, such as laminin and collagen type $\mathrm{IV}$, for axonal growth, and produce neurite promoting factors to guide the growing axon $[13,14]$. Thus, the aim of the present study was to investigate whether a hyaluronan-gelatin electrospun matrix has potential as a neurograft for peripheral nerve repair.

\section{Materials and Methods}

2.1. Reagents. Dulbecco's Modified Eagle Medium (DMEM), fetal bovine serum (FBS), and trypsin were purchased from GIBCO (Grand Island, NY, USA). Gelatin type A (Gel) was purchased from the Sigma-Aldrich Chemical Company (St. Louis, MO, USA). Sodium hyaluronan was purchased from NovaMatrix (Drammen, Norway). The RT4-D6P2T rat Schwann cells were purchased from BCRC (Bioresource Collection and Research Center, Taiwan). The other chemicals used were of reagent grade unless otherwise stated.
2.2. Preparation of Hyaluronan-Gelatin Composite Nanofibrous Matrix (HA-Gel). To allow complete evaporation of the solvent, the solvent used in this study was a mixture of formic acid and 1,1,1,3,3,3-hexafluoro-2-propanol (HFIP) at a volume/volume ratio of $3 / 7$. The gelatin powder was dissolved in the solvent mixture to a concentration of $12 \%(\mathrm{w} / \mathrm{v})$. Various concentrations of sodium hyaluronan $(0 \%, 0.5 \%, 1 \%$, and $1.5 \%$ $\mathrm{w} / \mathrm{v})$ were added to the gelatin solution and dissolved by vortexing until the solution was clear. For the electrospinning process, the polymer solution was placed in a plastic syringe fitted with an $18 \mathrm{G}$ needle and attached to a syringe pump that provided a steady flow rate of $8.5 \mu \mathrm{L} / \mathrm{min}$. The electrospinning voltage of $2.3 \mathrm{kV} / \mathrm{cm}$ was applied using a high-voltage power supply (Glassman High Voltage, Inc.).

2.3. Characterization of the Electrospun Nanofibers. The fiber morphology and diameter of the HA-Gels were determined by a field emission scanning electron microscope (SEM; model S-4800, Hitachi, Tokyo, Japan). Briefly, the electrospun matrices were sputter-coated with gold and visualized by SEM at an accelerating voltage of $5 \mathrm{kV}$. The fiber diameter was analyzed using image analysis (ImageJ software 1.42, National Institutes of Health, USA). The average fiber diameter and standard deviation were calculated from 100 random measurements.

2.4. Cell Culture on HA-Gel. Because the HA-Gels readily dissolved in aqueous media, it was necessary to cross-link them before using them for cell culture. The electrospun matrices were cross-linked by treatment with $\mathrm{N}$-(3-dimethylaminopropyl)-N-ethylcarbodiimide hydrochloride (EDC). The matrix was immersed in 1\% EDC solution in 95\% ethanol for $2 \mathrm{hrs}$ and then washed repeatedly with $0.01 \mathrm{M}$ PBS to remove the residual EDC. Finally, the electrospun matrices were sterilized by exposure to ultraviolet light in a sterile hood overnight. The electrospun matrices were placed in 24-well tissue culture plates containing a suspension of RT4-D6P2T rat Schwann cells (BCRC no. 60508) $\left(5 \times 10^{4}\right.$ cells/well) in DMEM supplemented with $10 \%$ (v/v) FBS, $100 \mathrm{U} / \mathrm{mL}$ of penicillin, and $100 \mu \mathrm{g} / \mathrm{mL}$ of streptomycin. The cultures of cellseeded electrospun matrices were harvested at 1, 2, 3, and 4 hrs for cell attachment assays and on days 1, 3, and 6 days for cell proliferation assays. Cell viability was determined using the MTT assay. At each time point, three samples were used to measure the number of cells attached to the electrospun matrices. The experiments were performed in triplicate.

2.5. Fluorescent Staining of the Cytoskeleton. The morphology of cells with their F-actin cytoskeleton fluorescently stained with fluorescein isothiocyanate (FITC)-conjugated phalloidin and nuclei stained with DAPI was examined. After 1 or 4 hrs of cell culture, cells were fixed with $3.7 \%(\mathrm{v} / \mathrm{v})$ paraformaldehyde in PBS for $10 \mathrm{~min}$, followed by 2 times washing with PBS and permeabilization with $0.1 \%$ (v/v) Triton X-100 in PBS for $5 \mathrm{~min}$. After washing with PBS, the samples were blocked with $1 \%$ bovine serum albumin in PBS for $1 \mathrm{hr}$. After blocking, the samples were stained with $6.4 \mu \mathrm{M}$ FITCconjugated phalloidin for $20 \mathrm{~min}$. Cell nuclei were stained 
TABLE 2: Sample notation, average diameter of hyaluronan-gelatin composite nanofibers.

\begin{tabular}{lcccc}
\hline $\begin{array}{l}\text { Sample } \\
\text { notation }\end{array}$ & $\begin{array}{c}\text { Gelatin concentration } \\
(\mathrm{mg} / \mathrm{mL})\end{array}$ & $\begin{array}{c}\text { Hyaluronan } \\
\text { concentration }(\mathrm{mg} / \mathrm{mL})\end{array}$ & $\begin{array}{c}\text { Average diameter } \\
\text { Standard deviation }(\mathrm{nm})\end{array}$ & $\begin{array}{c}\text { 1st quartile; 3rd quartile } \\
(\mathrm{nm})\end{array}$ \\
\hline Gel & 12 & 0 & $130 \pm 30$ & $109 ; 144$ \\
0.5 HA-Gel & 12 & 0.5 & $294 \pm 87$ & $230 ; 352$ \\
1 HA-Gel & 12 & 1 & $362 \pm 129$ & $282 ; 422$ \\
1.5 HA-Gel & 12 & 1.5 & $224 \pm 54$ & $179 ; 256$ \\
\hline
\end{tabular}

with 4',6-diamidino-2-phenylindole (DAPI). After staining, the samples were washed with PBS and observed under a fluorescence microscope (TS100, Nikon) and a laser scanning confocal microscope (LSCM, Zeiss LSM 510 META).

2.6. Quantitative Real-Time PCR. Real-time PCR was used to determine the levels of neuregulin 1 (Nrg1), S100, nerve growth factor (NGF), low-affinity nerve growth factor receptor (p75), glial fibrillary acidic protein (GFAP), and myelin protein zero (P0) mRNA and 18S ribosomal RNA (as the internal control). Table 1 shows the sequences of the oligonucleotides that were used as PCR primers. Briefly, the reaction is followed by the manufacture protocol of SYBR Green PCR Master Mix Kit (Protech SA-SQGLR-V2). The real-time PCR conditions for the promoter region were $15 \mathrm{mins}$ at $94^{\circ} \mathrm{C}$, $1 \mathrm{~min}$ at $62.5^{\circ} \mathrm{C}$, and $15 \mathrm{~s}$ at $94^{\circ} \mathrm{C}$. Real-time PCR reactions were performed using an iQ5 Gradient Real-Time PCR system (Bio-Rad). The levels of RNA expression were calculated using the $2^{-\Delta \Delta \mathrm{Ct}}$ method.

2.7. Immunoblotting Analysis. Cells were seeded on substrates at $3 \times 10^{4} \mathrm{cell} / \mathrm{s} / \mathrm{cm}^{2}$ in medium. Immunoblotting to detect the Schwann cell-specific proteins neuregulin 1 (Nrgl), myelin protein zero (P0), and CD44 was performed after day 3 and day 6 of culturing. Cells were collected and lysed in lysis buffer. The supernatants were obtained by centrifugation at $4^{\circ} \mathrm{C}, 15,000 \times \mathrm{g}$ for $10 \mathrm{~min}$. The concentration of protein was analyzed using a Bradford Coomassie assay. The proteins $(30 \mu \mathrm{g} / \mu \mathrm{L})$ were fractionated by electrophoresis and electrotransferred to polyvinylidene difluoride film (PVDF). Blocking was performed using $5 \%(\mathrm{w} / \mathrm{v})$ nonfat milk, and then the primary antibody was applied to the membrane overnight at $4^{\circ} \mathrm{C}$. Antibodies specific to CD44 (Santa Cruz BiotechnologyINC. sc-9960), Nrgl (Santa Cruz Biotechnology-INC. sc28916), P0 (Santa Cruz Biotechnology-INC. sc-18531), and nucleophosmin B23 (Invitrogen 325200) were used. After incubation with a HRP-conjugated secondary antibody, the immunoreactive bands were detected using enhanced chemiluminescence detection (Millipore WBKLS0500). The immunoreactive bands were analyzed by ImageJ software (National Institutes of Health, USA). Nucleophosmin B23 was used as the internal control.

2.8. Statistical Analyses. Statistical analyses were performed using SPSS v.11 software. For each condition, the diameters of at least 100 randomly chosen fibers were measured. The fiber diameters were analyzed using a one-way ANOVA with Tukey's post hoc test. The cell viability, gene expression, and immunoblotting were analyzed with the nonparametric Mann-Whitney $U$ test. Differences at $P<0.05$ were considered statistically significant.

\section{Results}

3.1. Fabrication of Hyaluronan-Gelatin Electrospun Nanofibers. Formic acid is a good solvent for hyaluronan and gelatin. However, gelatin and hyaluronan/gelatin which dissolved in a formic acid solution cannot be fabricated into nanofibrous matrices by electrospinning. In a previous study, hexafluoropropanol was found to be a suitable solvent to dissolve hyaluronan/collagen [15]. To obtain hyaluronan-gelatin nanofibers (HA-Gel), we used the cosolvent method by mixing formic acid and HFIP.

Figure 1 shows that the synthetic product was a three-dimensional nonwoven nanofibrous hyaluronan-gelatin matrix with interconnected pores. The fiber diameters were $130 \pm$ $30 \mathrm{~nm}, 294 \pm 87 \mathrm{~nm}, 362 \pm 129 \mathrm{~nm}$, and $224 \pm 54 \mathrm{~nm}$ for Gel, 0.5 HA-Gel, $1 \mathrm{HA}-\mathrm{Gel}$, and 1.5 HA-Gel, respectively. These dimensions are similar to those of the native fibrous proteins in the ECM. We found that the diameters of the fibers in the HA-Gel matrix were significantly different from those in the Gel matrix $(P<0.05$, one-way ANOVA with Tukey's post hoc test) (Table 2). In addition, the diameters of the fibers gradually increased and then decreased as the concentration of hyaluronan was gradually increased.

The Gel and HA-Gel fibers cross-linked with EDC are shown in Figure 2. The Gel and HA-Gel fibers still maintained their morphology. However, the fibers appeared to be more rubbery and were fused at fiber junctions.

3.2. Cell Attachment and Proliferation. Cellular functions, including spreading, proliferation, and differentiation, are sensitive to the composition and surface topography of the matrix. To test the effects of the hyaluronan/gelatin weight ratios of the nanofibrous matrix on cell morphology, we incubated the RT4-D6P2T rat Schwann cells on hyaluronan and gelatin matrices with different weight ratios. The morphologies of the Schwann cells that adhered to the different matrices were investigated by staining the actin cytoskeleton with FITC-phalloidin and visualizing the cells with a fluorescence microscope (shown in Figure 3) and a laser scanning confocal microscope (shown in Figure 4). We found that most of the Schwann cells cultured on the HA-Gel matrix appeared to have spread during the $4 \mathrm{hr}$ attachment period. However, it was obvious that the cells cultured on the Gel matrix remained round after $4 \mathrm{~h}$ (Figure 3(e) and Figure 4(e)). The attachment and proliferation of the Schwann cells on the Gel 


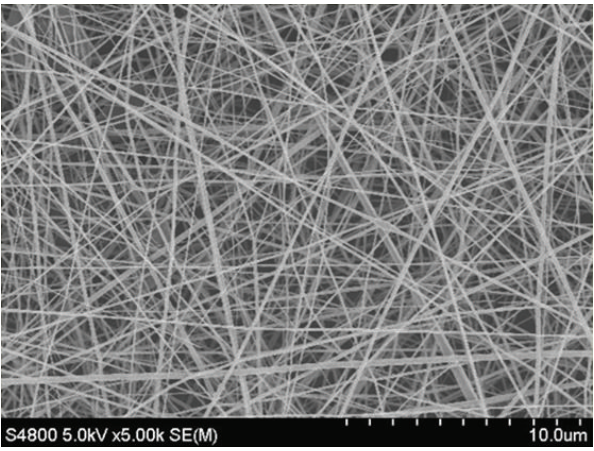

(a)

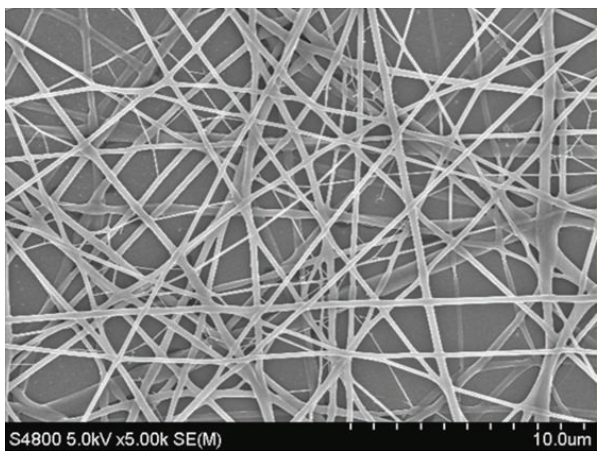

(c)

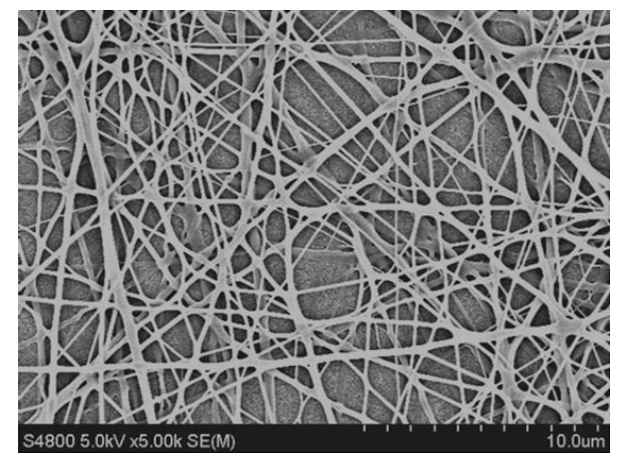

(b)

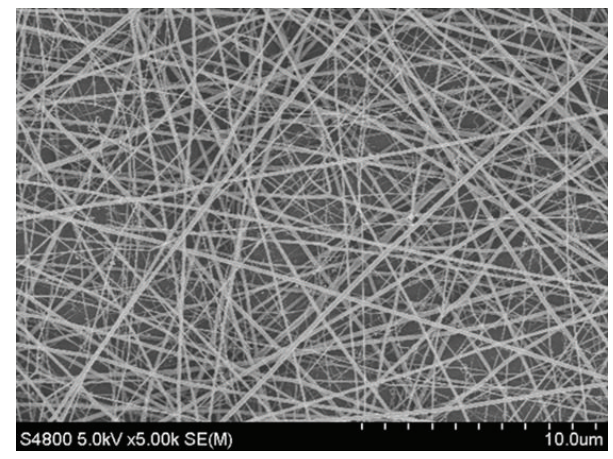

(d)

FIGURE 1: Scanning electron microscopy images of as-electrospun HA-Gel matrix at a magnification of 5000x. (a) Gel, (b) $0.5 \mathrm{HA}-\mathrm{Gel}$, (c) $1 \mathrm{HA}-$ Gel, and (d) 1.5 HA-Gel. The scale bar represents $10 \mu \mathrm{m}$.

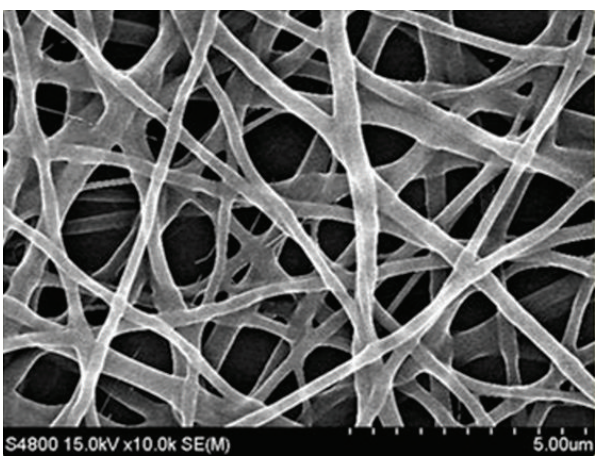

(a)

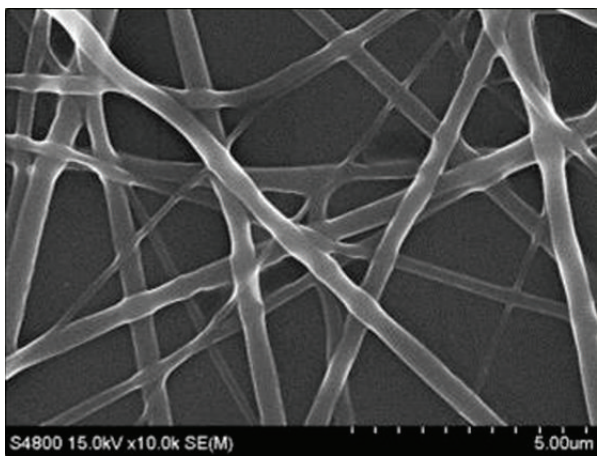

(c)

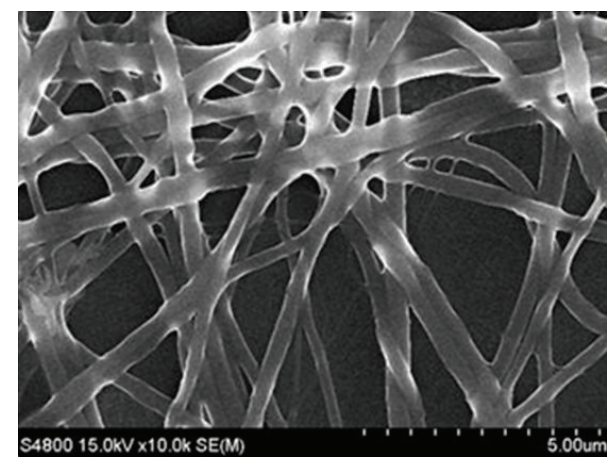

(b)

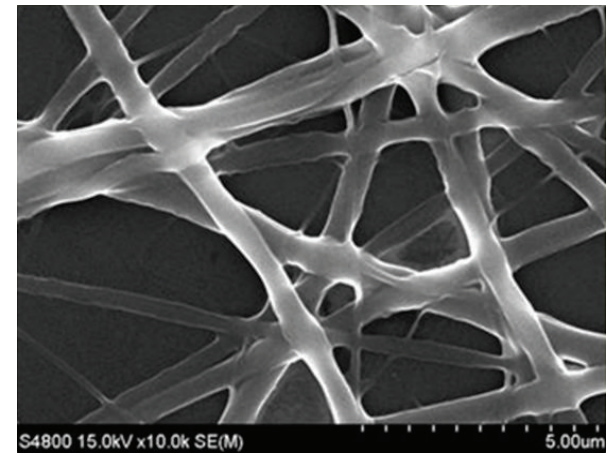

(d)

FIGURE 2: Scanning electron microscopy images of electrospun HA-Gel matrix after EDC treatment at a magnification of 10000x. (a) Gel, (b) $0.5 \mathrm{HA}-\mathrm{Gel}$, (c) $1 \mathrm{HA}-\mathrm{Gel}$, and (d) $1.5 \mathrm{HA}-\mathrm{Gel}$. The scale bar represents $5 \mu \mathrm{m}$. 


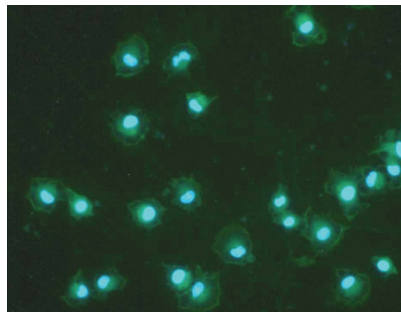

(a)



(e)

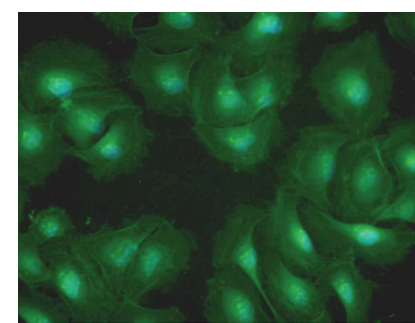

(b)

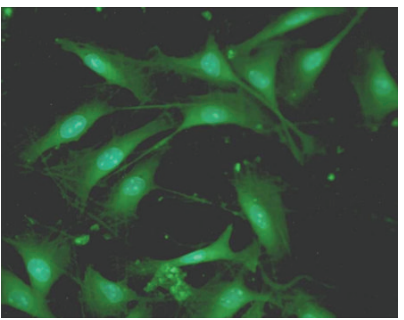

(f)

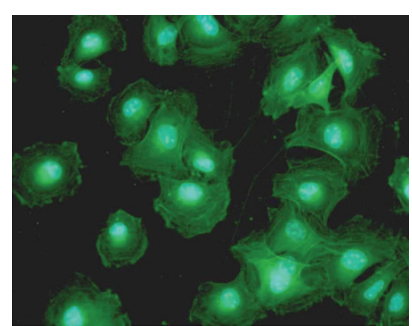

(c)

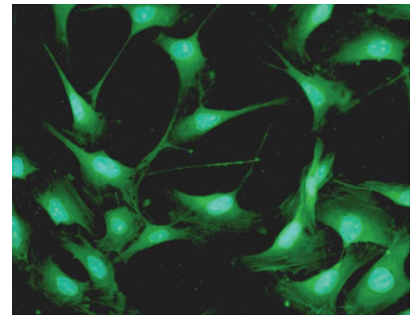

(g)

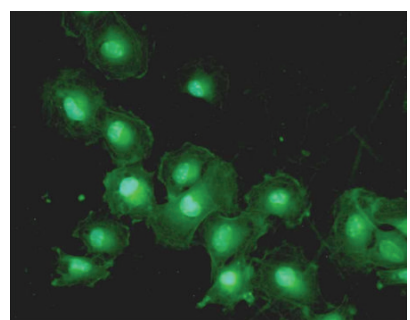

(d)

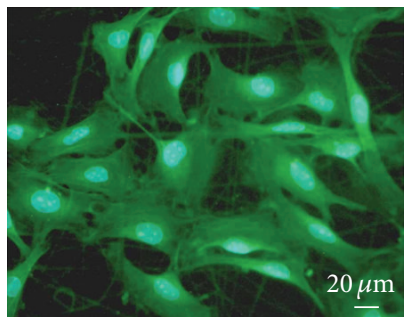

(h)

FIGURE 3: Fluorescence micrographs of F-actin stained RT4-D6P2T Schwann cell grown on an electrospun matrix of Gel ((a), (e)), 0.5 HA-Gel ((b), (f)), 1 HA-Gel ((c), (g)), and 1.5 HA-Gel ((d), (h)) for $1 \mathrm{hr}((\mathrm{a}),(\mathrm{b}),(\mathrm{c})$, and (d)) or 4 hrs ((e), (f), (g), and (h)). Cytoskeletal F-actin was stained green with FITC, and cell nuclei were stained blue with DAPI. (Scale bar $=20 \mu \mathrm{m}$.)

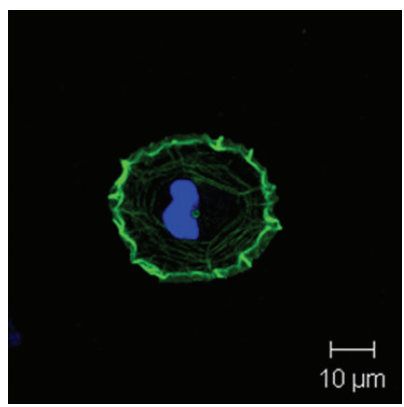

(a)

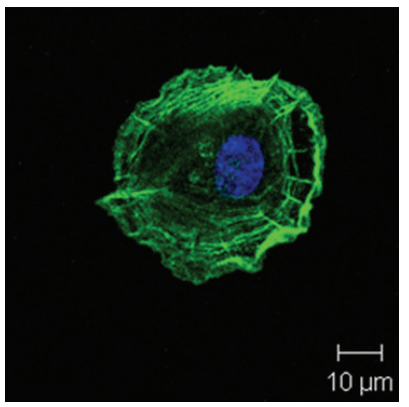

(e)

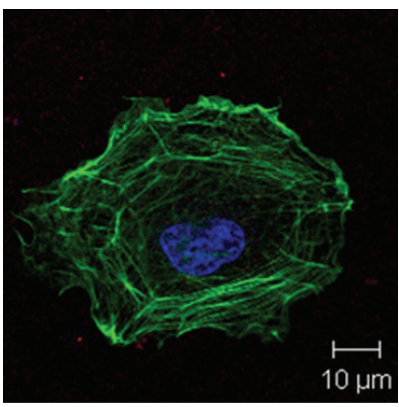

(b)

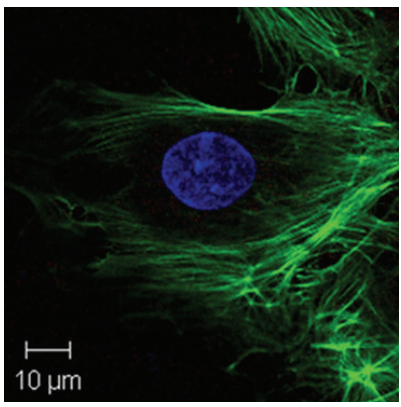

(f)

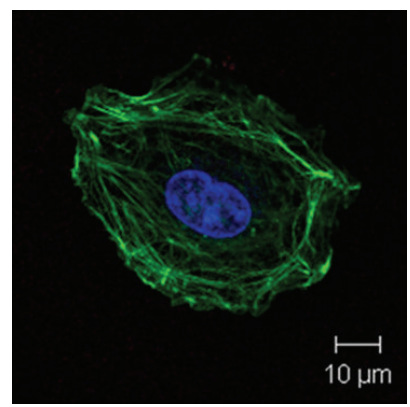

(c)

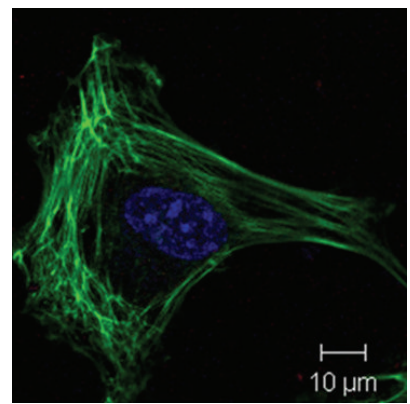

(g)

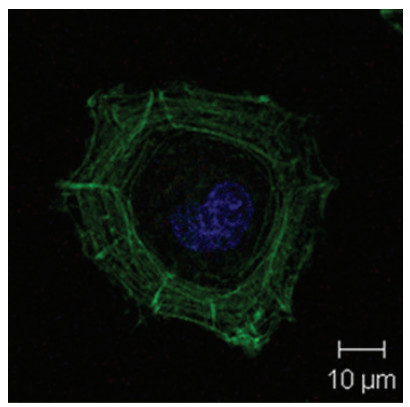

(d)

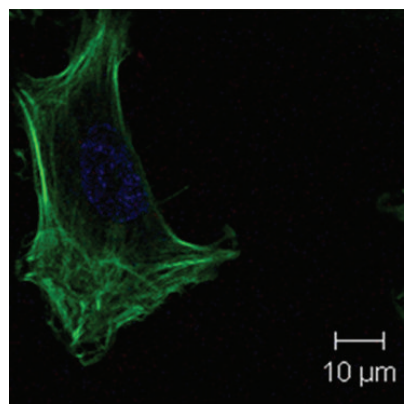

(h)

Figure 4: Confocal micrographs of F-actin stained RT4-D6P2T Schwann cell on an electrospun matrix of Gel ((a), (e)), 0.5 HA-Gel ((b), (f)), 1 HA-Gel ((c), (g)), and 1.5 HA-Gel ((d), (h)) for 1 hour ((a), (b), (c), and (d)) and 4 hours ((e), (f), (g), and (h)). Cytoskeletal F-actin was stained green with FITC, and cell nuclei were stained blue with DAPI. (Scale bar $=10 \mu \mathrm{m}$.)

and HA-Gel matrices were quantified using the MTT assay. There were no significant differences in attachment or proliferation among the cells cultured on 0.5 HA-Gel, $1 \mathrm{HA}-\mathrm{Gel}$, or 1.5 HA-Gel $(P>0.05)$ (as shown in Figure 5).

3.3. Real-Time PCR Analysis. The expression levels of Schwann cell-specific genes were analyzed using real-time
PCR for Nrg1, S100, NGF, p75, GFAP, and P0 and 18S ribosomal RNA (as shown in Figure 6). On day 3, the Schwann cells displayed insignificantly increased gene expression levels of Nrg1, S100, NGF, p75, GFAP, and P0 when grown on $0.5 \mathrm{HA}-$ Gel, 1 HA-Gel, or 1.5 HA-Gel relative to those obtained from cells grown on Gel $(P>0.05)$. However, on day 6, the Schwann cells showed significantly higher gene expression 


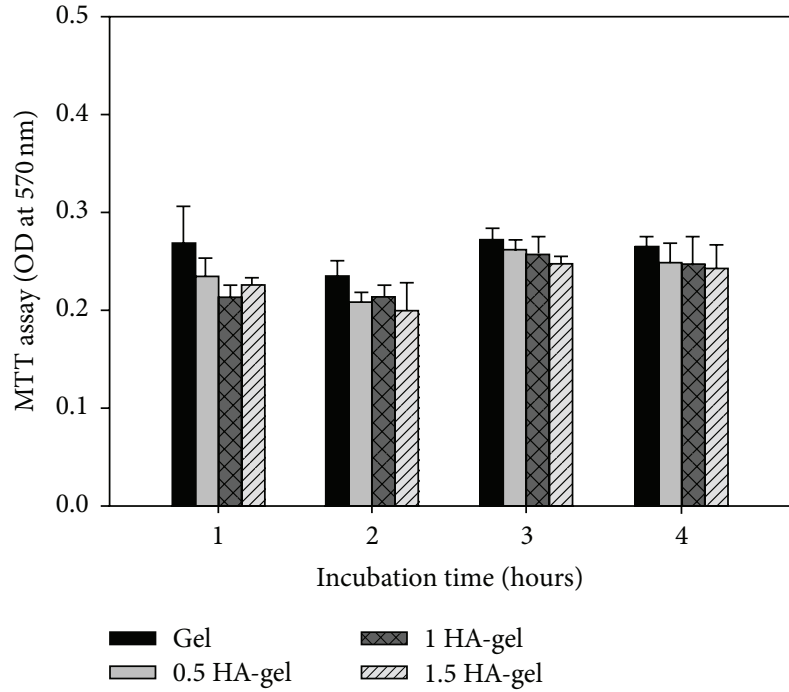

(a)

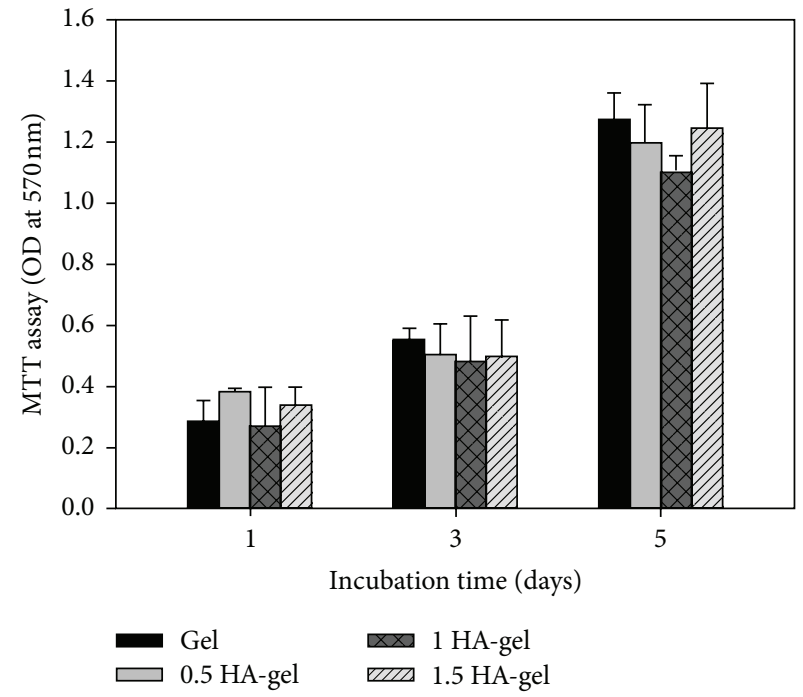

(b)

FIGURE 5: Quantification of RT4-D6P2T Schwann cells on Gel and HA-Gel matrices. The MTT assay was used to quantify cell attachment and proliferation on Gel and HA-Gel. (a) The attachment of RT4-D6P2T Schwann cells on various matrices after culturing for up to 4 hrs. (b) The viability of RT4-D6P2T Schwann cells on various matrices after culturing for up to 6 days. The data are presented as the means \pm SD, $n=4$. No statistically significant differences in cell attachment or proliferation on the HA-Gel and Gel were found.

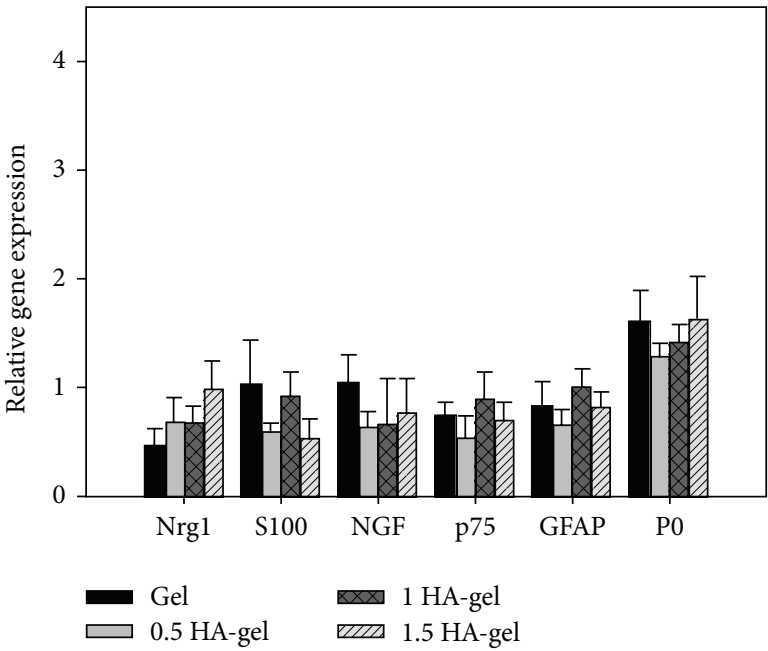

(a)

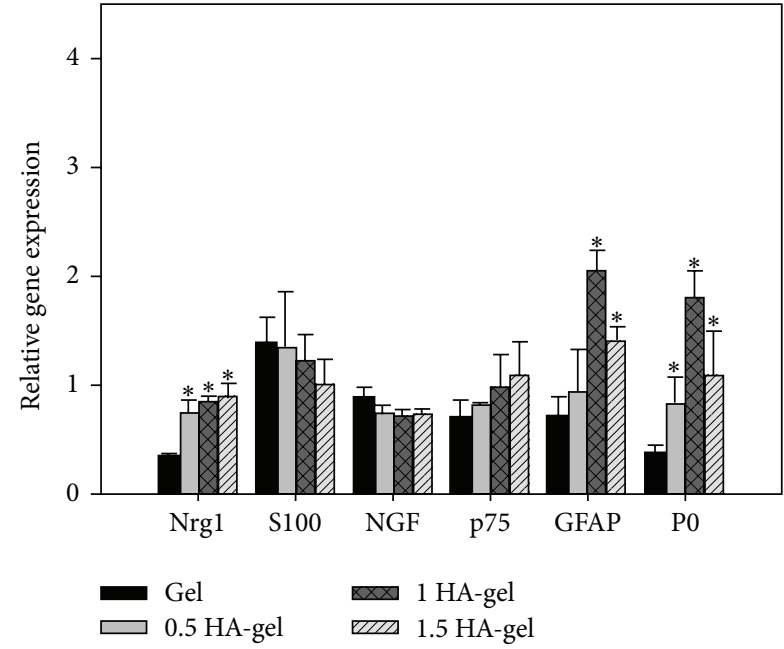

(b)

FIGURE 6: Real-time PCR analyses of the nerve-associated genes expressed by RT4-D6P2T Schwann cells on various matrices after culturing for (a) 3 days or (b) 6 days. The data are shown as the fold change relative to those obtained from cells grown on the culture dish surface. The data are presented as the means $\pm \mathrm{SD}(n=4)$. $\left(^{*}\right)$ denotes significant difference between HA-Gel and Gel $(P<0.05)$.

levels of Nrg1, GFAP, and P0 when grown on 0.5 HA-Gel, 1 HA-Gel, or 1.5 HA-Gel compared to cells grown on Gel $(P<0.05)$ (as shown in Figure 6).

3.4. Western Blot Analysis. The CD44 levels expressed on day 3 and day 6 by the RT4-D6P2T rat Schwann cells grown on the different matrices were not significantly different $(P>$ 0.05 ). The levels of $\mathrm{Nrgl}$ and $\mathrm{P} 0$ were higher in the cells grown on 0.5 HA-Gel, 1 HA-Gel, or 1.5 HA-Gel than in the cells grown on Gel on day $6(P<0.05)$ (as shown in Figure 7).
However, the levels of Nrg1 and P0 on day 3 and day 6 were not significantly different among the cells grown on $0.5 \mathrm{HA}-$ Gel, 1 HA-Gel, or 1.5 HA-Gel $(P>0.05)$.

\section{Discussion}

Sponge forms of gelatin, hyaluronan, and their composites have been widely used to fabricate scaffolds for tissue engineering [16-18]. However, the physical structure of the sponge form of these matrices is not acceptable for the tissue 


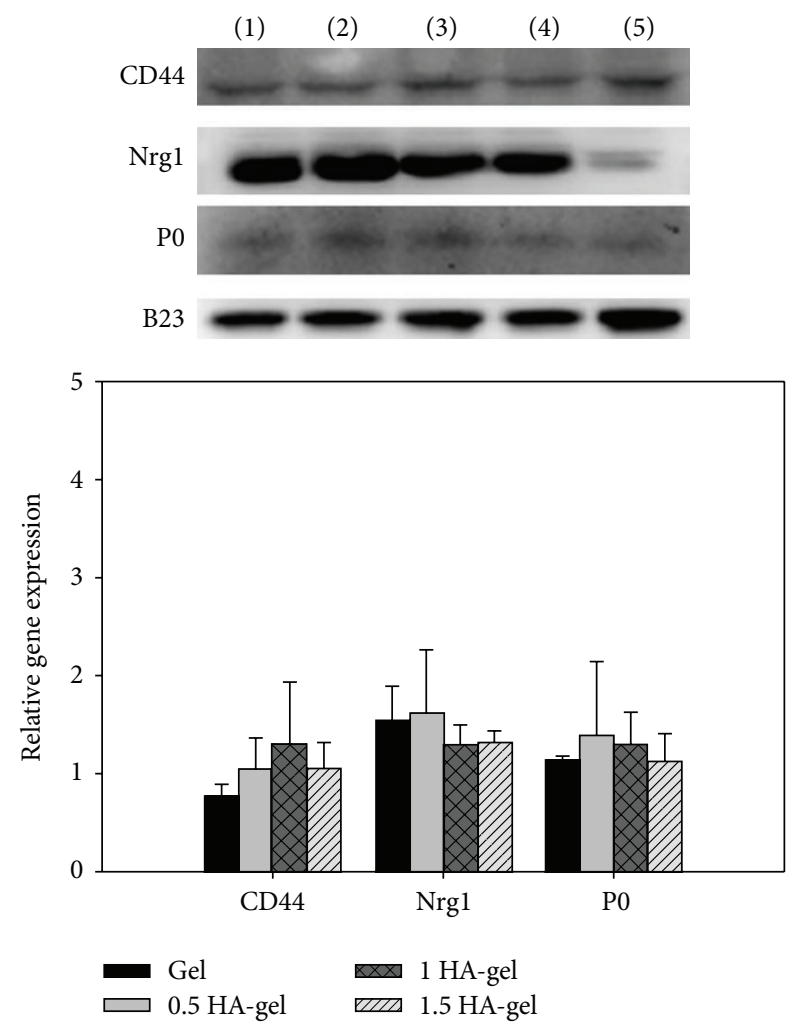

(a)
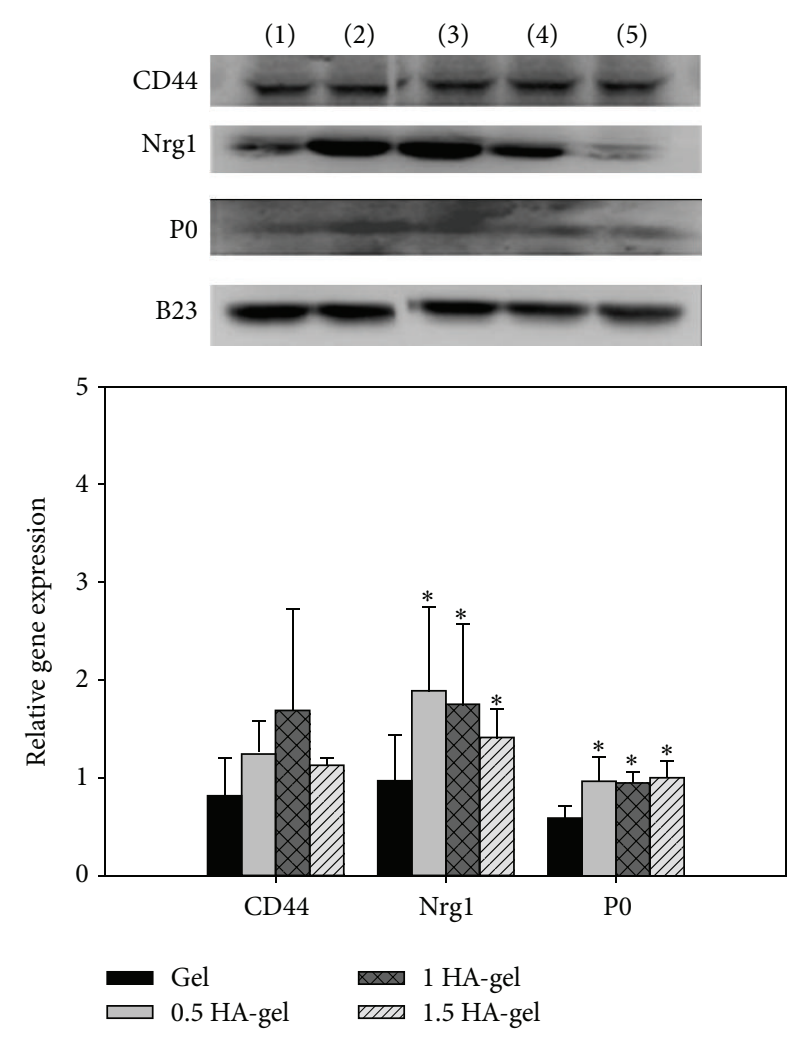

(b)

FIGURE 7: Western blot analysis of CD44, Nrg1, and P0 proteins in RT4-D6P2T Schwann cells cultured for (a) 3 or (b) 6 days. Densitometric quantification of the protein bands was performed by normalizing with B23. The data are shown as the fold change relative to those obtained from cells grown on the culture dish surface. The data are presented as the means $\pm \operatorname{SD}(n=3)$. $\left({ }^{*}\right)$ denotes significant difference between HA-Gel and Gel $(P<0.05)$. (1) Gel, (2) 0.5 HA-Gel, (3) $1 \mathrm{HA}-\mathrm{Gel}$, (4) $1.5 \mathrm{HA}-\mathrm{Gel}$, and (5) culture dish.

regeneration. A porous and nanofibrous biodegradable matrix that mimics a natural ECM is the optimal scaffold for tissue engineering [19]. It is widely accepted that cell adhesion and most cellular activities, including spreading, migration, proliferation, gene expression, and cytoskeletal function, are sensitive to the nanotopography [20] and molecular composition of the matrix [15]. Xu et al. demonstrated that there were stronger interactions between the cells and the nanofibers [21]. Consequently, most researchers believe that a nanofibrous matrix may promote cell growth more effectively than a sponge substrate.

Several methods have been used to fabricate the fibrous matrix, but the electrospinning is a simple and effective fabrication technique to produce nano-/microfibers. The morphology and diameter of electrospun fibers are dependent on many processing parameters, including the molecular weight of the polymer, the concentration (or viscosity), the conductivity, the applied voltage, and the feeding rate of the polymer solution. In our previous study, we found that increasing the concentration of hyaluronan would increase the charge density on the surface of the electrospinning jet, which would induce higher electrostatic forces and result in the formation of smaller diameter fibers [15]. On the other hand, the diameter of the electrospun fibers increases with increasing concentrations of a polymer solution. A combination of the aforesaid factors might explain why the mean fiber diameters increased at lower hyaluronan concentrations and decreased at higher hyaluronan concentrations.

Hyaluronan is found in all vertebrate tissues and is important for the organization of pericellular and extracellular matrices [22]. Hyaluronan plays a crucial role in tissue hydration and lubrication and in the regulation of cell functions,including cell attachment, cell mitosis, cell migration, tumor development, metastasis, cell apoptosis, and inflammation $[23,24]$. The function of hyaluronan depends upon its interaction with the cell surface receptors, including the receptor for hyaluronan-mediated motility (RHAMM), cluster determinant 44 (CD44), lymphatic vessel endothelial hyaluronan receptor (LYVE-1), hyaluronan receptor for endocytosis (HARE), liver endothelial cell clearance receptor (LEC receptor), and toll-like receptor 4 (TLR4) $[25,26]$. Hyaluronan has been shown to influence the interaction of glial cells with axons, to reduce the formation of scar tissue and to provide a suitable environment for tissue repair [27-29]. Wang et al. found that hyaluronan played a vital role in the biological processes involved in the deposition and remodeling of the fibrin extracellular matrix to increase the level of myelination of axons after 4 weeks in vivo [30]. Sherman noted that the CD44 transmembrane glycoprotein plays a key role in Schwann cellneuron interaction. The reduction of CD44 expression 
in vitro decreased Schwann cell-neurite adhesion and caused the apoptosis of Schwann cells [31].

The peripheral nerve repair process depends on Schwann cells, which synthesize and secrete important substances such as neuregulin-1 (Nrg1), GFAP, and P0. Nave proposed that Nrg1 is essential for every developmental stage of Schwann cells and for the myelination of axons [32]. Nrg1 is an epidermal growth factor-like ligand that interacts with the tyrosine kinase ErbB receptor to regulate many aspects of neural development. Moreover, Ghatak et al. demonstrated that ErbB2 can be activated by HA through CD44-mediated mechanisms to promote the proliferation and differentiation of Schwann cells [33].

GFAP is an intermediate filament protein that functions as a scaffold for cytoskeletal assembly and maintenance [34]. GFAP also plays a vital role in Schwann cell proliferation and the upregulation of Schwann cell-specific cytoskeletal constituents after nerve damage [35]. Myelin protein zero (P0) is the major adhesive and structural protein of the myelin sheath of peripheral nerves [36]. P0 is expressed by myelinating Schwann cells and is necessary for normal myelin structure and function [37]. Some studies have demonstrated that P0 promoted the regeneration of injured axons [38]. From the results of real-time Q-PCR and western blotting, we found that Nrgl, GFAP, and P0 gene expression and Nrgl and P0 protein expression were enhanced at day 6 when Schwann cells were cultured on HA-Gel. We speculate that the HA-Gel enhanced myelination via the interaction of the surface CD44 receptors of Schwann cells with hyaluronan.

\section{Conclusion}

Schwann cells grown on hyaluronan-gelatin nanofibrous matrices showed better organized F-actin stress fibers, higher levels of Nrg1, GFAP, and P0 mRNA, and higher levels of Nrg1 and P0 protein compared to those grown on the gelatin nanofibrous matrix. These findings suggest that the hyaluronan-gelatin nanofibrous matrix could potentially be used in the repair of injured peripheral nerves.

\section{Conflict of Interests}

The authors declare that there is no conflict of interests regarding the publication of this paper.

\section{References}

[1] T. A. Ferguson and Y. J. Son, "Extrinsic and intrinsic determinants of nerve regeneration," Journal of Tissue Engineering, vol. 2, no. 1, 2011.

[2] C. E. Schmidt and J. B. Leach, "Neural tissue engineering: strategies for repair and regeneration," Annual Review of Biomedical Engineering, vol. 5, pp. 293-347, 2003.

[3] T. E. Trumble and F. G. Shon, "The physiology of nerve transplantation," Hand Clinics, vol. 16, no. 1, pp. 105-122, 2000.

[4] R. E. Gámez Sazo, K. Maenaka, W. Gu, P. M. Wood, and M. B. Bunge, "Fabrication of growth factor- and extracellular matrixloaded, gelatin-based scaffolds and their biocompatibility with
Schwann cells and dorsal root ganglia," Biomaterials, vol. 33, no. 33, pp. 8529-8539, 2012.

[5] J. M. Medina, A. Thomas, and C. R. Denegar, "Knee osteoarthritis: should your patient opt for hyaluronic acid injection?" Journal of Family Practice, vol. 55, no. 8, pp. 669-675, 2006.

[6] E. Nyman, F. Huss, T. Nyman, J. Junker, and G. Kratz, "Hyaluronic acid, an important factor in the wound healing properties of amniotic fluid: in vitro studies of re-epithelialisation in human skin wounds," Journal of Plastic Surgery and Hand Surgery, vol. 47, no. 2, pp. 89-92, 2013.

[7] G. Y. Özgenel, "Effects of hyaluronic acid on peripheral nerve scarring and regeneration in rats," Microsurgery, vol. 23, no. 6, pp. 575-581, 2003.

[8] J. Taipale and J. Keski-Oja, "Growth factors in the extracellular matrix," FASEB Journal, vol. 11, no. 1, pp. 51-59, 1997.

[9] F. Berthiaume, P. V. Moghe, M. Toner, and M. L. Yarmush, "Effect of extracellular matrix topology on cell structure, function, and physiological responsiveness: hepatocytes cultured in a sandwich configuration," FASEB Journal, vol. 10, no. 13, pp. 1471-1484, 1996.

[10] C. Li, C. Vepari, H.-J. Jin, H. J. Kim, and D. L. Kaplan, "Electrospun silk-BMP-2 scaffolds for bone tissue engineering," Biomaterials, vol. 27, no. 16, pp. 3115-3124, 2006.

[11] M. G. Burnett and E. L. Zager, "Pathophysiology of peripheral nerve injury: a brief review," Neurosurgical Focus, vol. 16, no. 5, article E1, 2004.

[12] S. S. Scherer and J. Salzer, "Axon-Schwann cell interaction during peripheral nerve degeneration and regeneration," in Glial Cell Development, pp. 299-330, Oxford University Press, London, UK, 2003.

[13] J. Hu, J. Zhou, X. Li, F. Wang, and L. Hezuo, "Schwann cells promote neurite outgrowth of dorsal root ganglion neurons through secretion of nerve growth factor," Indian Journal of Experimental Biology, vol. 49, no. 3, pp. 177-182, 2011.

[14] A. I. Gravvanis, A. A. Lavdas, A. Papalois, D. A. Tsoutsos, and R. Matsas, "The beneficial effect of genetically engineered Schwann cells with enhanced motility in peripheral nerve regeneration: review," Acta Neurochirurgica, vol. 100, pp. 51-56, 2007.

[15] F.-Y. Hsu, Y.-S. Hung, H.-M. Liou, and C.-H. Shen, "Electrospun hyaluronate-collagen nanofibrous matrix and the effects of varying the concentration of hyaluronate on the characteristics of foreskin fibroblast cells," Acta Biomaterialia, vol. 6, no. 6, pp. 2140-2147, 2010.

[16] L. Hong, I. Peptan, P. Clark, and J. J. Mao, "Ex vivo adipose tissue engineering by human marrow stromal cell seeded gelatin sponge," Annals of Biomedical Engineering, vol. 33, no. 4, pp. 511517, 2005.

[17] Y. C. Chen, W. Y. Su, S. H. Yang, A. Gefen, and F. H. Lin, "In situ forming hydrogels composed of oxidized high molecular weight hyaluronic acid and gelatin for nucleus pulposus regeneration," Acta Biomaterialia, vol. 9, no. 2, pp. 5181-5193, 2013.

[18] Y. Liu, X. Z. Shu, S. D. Gray, and G. D. Prestwich, "Disulfidecrosslinked hyaluronan-gelatin sponge: growth of fibrous tissue in vivo," Journal of Biomedical Materials Research A, vol. 68, no. 1, pp. 142-149, 2004.

[19] H. M. Lin, Y. H. Lin, and F. Y. Hsu, "Preparation and characterization of mesoporous bioactive glass/polycaprolactone nanofibrous matrix for bone tissues engineering," Journal of Materials Science: Materials in Medicine, vol. 23, no. 11, pp. 2619-2630, 2012. 
[20] K. S. Park, K. J. Cha, I. B. Han et al., "Mass-producible nano-featured polystyrene surfaces for regulating the differentiation of human adipose-derived stem cells," Macromolecular Bioscience, vol. 12, no. 11, pp. 1480-1489, 2012.

[21] C. Xu, R. Inai, M. Kotaki, and S. Ramakrishna, "Electrospun nanofiber fabrication as synthetic extracellular matrix and its potential for vascular tissue engineering," Tissue Engineering, vol. 10, no. 7-8, pp. 1160-1168, 2004.

[22] T. Laurent, "The biology of hyaluronan. Introduction," Ciba Foundation Symposium, vol. 143, pp. 1-20, 1989.

[23] B. P. Toole and M. G. Slomiany, "Hyaluronan: a constitutive regulator of chemoresistance and malignancy in cancer cells," Seminars in Cancer Biology, vol. 18, no. 4, pp. 244-250, 2008.

[24] C. Cencetti, D. Bellini, C. Longinotti, A. Martinelli, and P. Matricardi, "Preparation and characterization of a new gellan gum and sulphated hyaluronic acid hydrogel designed for epidural scar prevention," Journal of Materials Science: Materials in Medicine, vol. 22, no. 2, pp. 263-271, 2011.

[25] J. B. Park, H.-J. Kwak, and S.-H. Lee, "Role of hyaluronan in glioma invasion," Cell Adhesion \& Migration, vol. 2, no. 3, pp. 202207, 2008.

[26] M. A. Solis, Y.-H. Chen, T. Y. Wong, V. Z. Bittencourt, Y.-C. Lin, and L. L. H. Huang, "Hyaluronan regulates cell behavior: a potential niche matrix for stem cells," Biochemistry Research International, vol. 2012, Article ID 346972, 11 pages, 2012.

[27] L. S. Sherman, T. A. Rizvi, S. Karyala, and N. Ratner, "CD44 enhances neuregulin signaling by Schwann cells," Journal of Cell Biology, vol. 150, no. 5, pp. 1071-1083, 2000.

[28] Y. T. Wei, W. M. Tian, X. Yu et al., "Hyaluronic acid hydrogels with IKVAV peptides for tissue repair and axonal regeneration in an injured rat brain," Biomedical Materials, vol. 2, no. 3, pp. S142-S146, 2007.

[29] Z. Z. Khaing and C. E. Schmidt, "Advances in natural biomaterials for nerve tissue repair," Neuroscience Letters, vol. 519, no. 2, pp. 103-114, 2012.

[30] K. K. Wang, I. R. Nemeth, and B. R. Seckel, "Hyaluronic acid enhances peripheral nerve regeneration in vivo," Microsurgery, vol. 18, no. 4, pp. 270-275, 1998.

[31] L. S. Sherman, T. A. Rizvi, S. Karyala, and N. Ratner, "CD44 enhances neuregulin signaling by Schwann cells," Journal of Cell Biology, vol. 150, no. 5, pp. 1071-1083, 2000.

[32] K.-A. Nave and J. L. Salzer, "Axonal regulation of myelination by neuregulin 1," Current Opinion in Neurobiology, vol. 16, no. 5, pp. 492-500, 2006.

[33] S. Ghatak, S. Misra, and B. P. Toole, "Hyaluronan constitutively regulates ErbB2 phosphorylation and signaling complex formation in carcinoma cells," Journal of Biological Chemistry, vol. 280, no. 10, pp. 8875-8883, 2005.

[34] P. A. Coulombe and P. Wong, "Cytoplasmic intermediate filaments revealed as dynamic and multipurpose scaffolds," Nature Cell Biology, vol. 6, no. 8, pp. 699-706, 2004.

[35] D. Triolo, G. Dina, I. Lorenzetti et al., "Loss of glial fibrillary acidic protein (GFAP) impairs Schwann cell proliferation and delays nerve regeneration after damage," Journal of Cell Science, vol. 119, no. 19, pp. 3981-3993, 2006.

[36] D. D’Urso, P. J. Brophy, S. M. Staugaitis et al., "Protein zero of peripheral nerve myelin: biosynthesis, membrane insertion, and evidence for homotypic interaction," Neuron, vol. 4, no. 3, pp. 449-460, 1990.

[37] D. M. Menichella, E. J. Arroyo, R. Awatramani et al., "Protein Zero is necessary for E-cadherin-mediated adherens junction formation in Schwann cells," Molecular and Cellular Neuroscience, vol. 18, no. 6, pp. 606-618, 2001.

[38] L. B. Spiryda, "Myelin protein zero and membrane adhesion," Journal of Neuroscience Research, vol. 54, no. 2, pp. 137-146, 1998. 

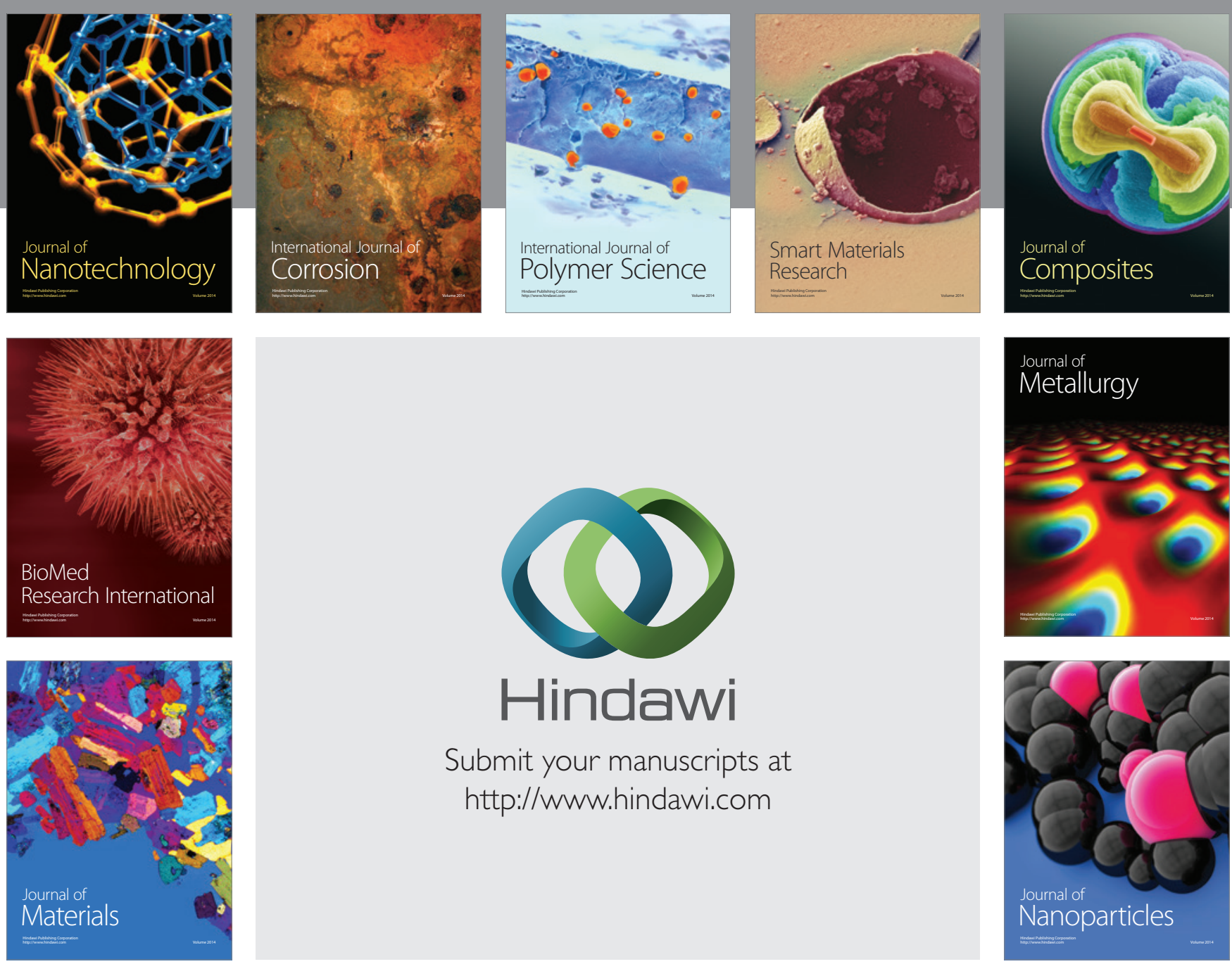

Submit your manuscripts at http://www.hindawi.com
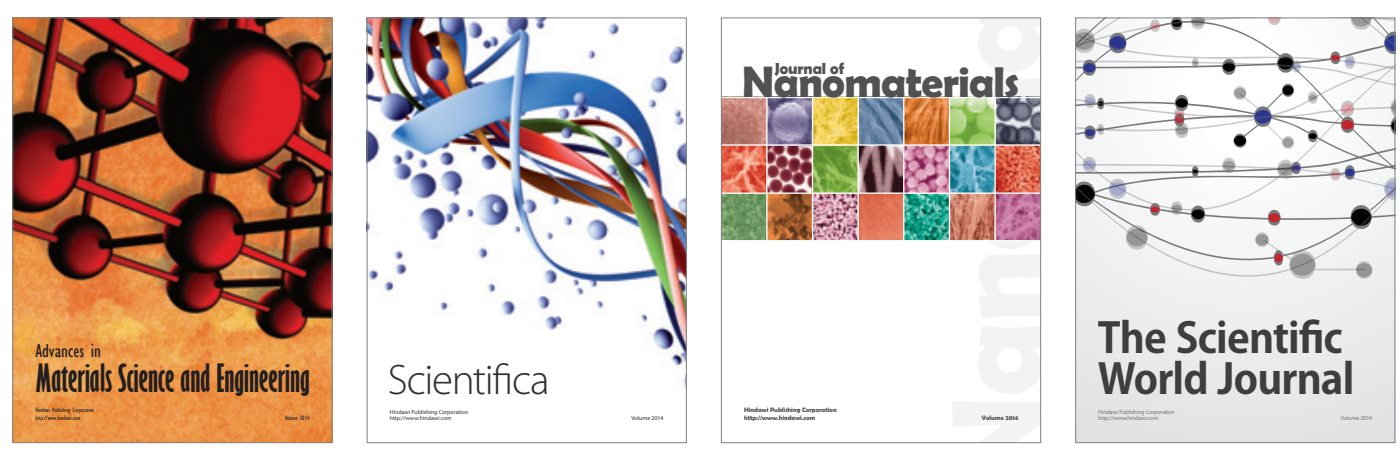

\section{The Scientific World Journal}
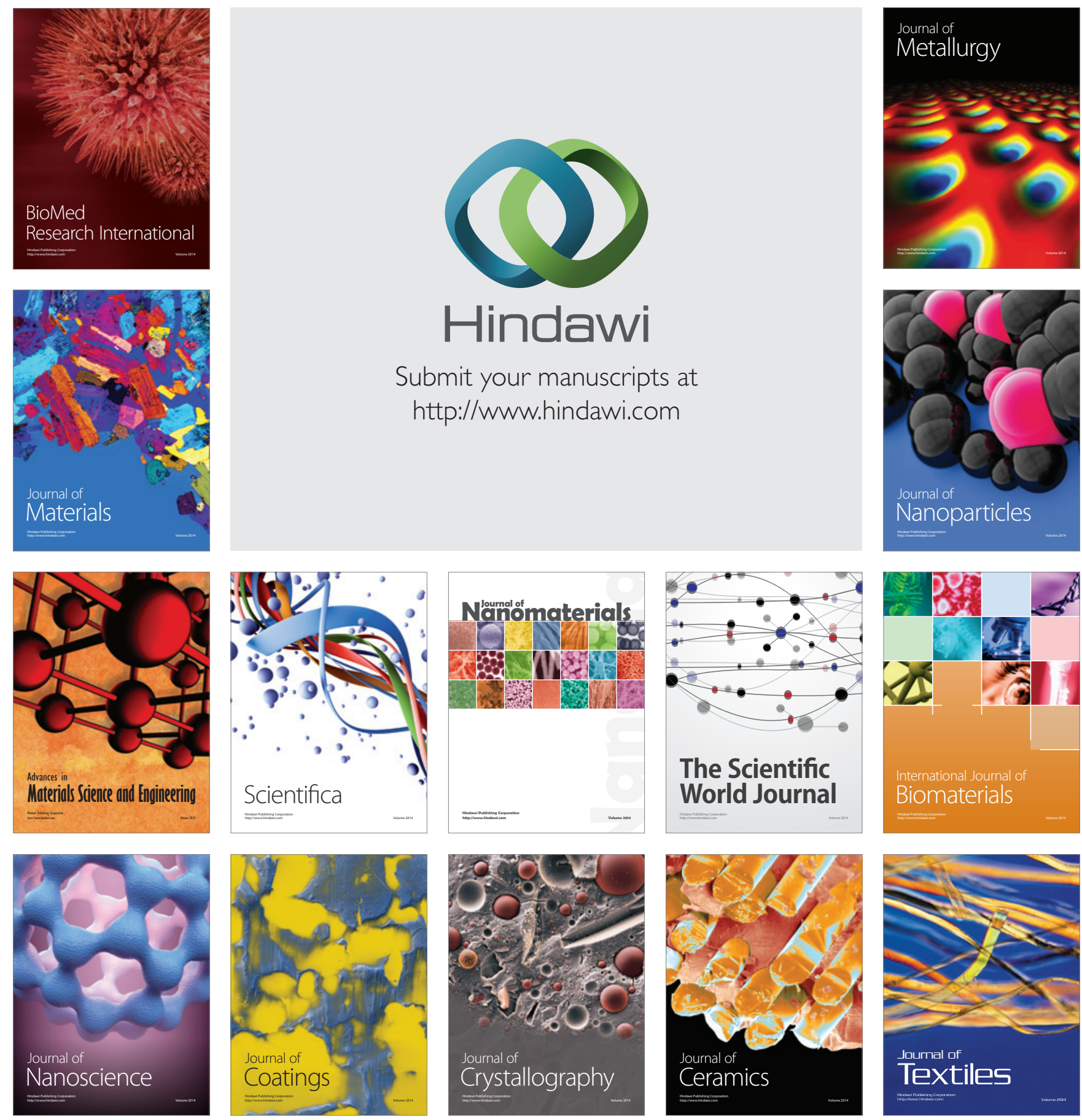